\title{
Development of robotics technologies in agriculture
}

\author{
Konstantin Mashkov ${ }^{1}$, Vasyliy Rubtsov ${ }^{2, *}$, and Ivan Rubtsov ${ }^{2}$ \\ ${ }^{1}$ Bauman Moscow State Technical University, Department of Multi-purpose caterpillar machines and \\ mobile robots, 105005 Moscow, Russia \\ ${ }^{2}$ Bauman Moscow State Technical University, Department of Robotic systems and mechatronics, \\ 105005, Moscow, Russia
}

\begin{abstract}
The assessment of the resource potential of agriculture of the Russian Federation was given. The main factors that determine a more complete use of the potential available in the industry were defined. The conditions for the introduction of modern technologies of "Precision farming" were described. The heterogeneous composition of the group maintenance of agricultural land was identified. The stages of development of robotics technologies in agriculture are considered. The problematic technology of unmanned agriculture was given. The socio-economic effects that can be achieved when implementing the proposed measures are described. The vector of development of agricultural robotics was given.
\end{abstract}

The analysis of the provisions of the strategy of sustainable development of rural areas of the Russian Federation for the period up to 2030 [1] shows the government's concern in the elimination of problems that impede the development of rural settlements.

It is recommended to the Agriculture Ministry of Russia and other interested Federal Executive authorities to increase the efficiency of planning of use of rural settlements:

- conducting an inventory of agricultural land;

- development of regional strategies for rural development settlements for the long-term period (until 2030), providing for including the involvement of agricultural land in circulation;

- creation of a unified Federal information system on the lander agricultural purposes, which provides for the contents of the relevant information about the boundaries of such lands, their square, on types of actual and permitted use, types of soils and types of lands, qualitative characteristics of agricultural lands purposes, as well as the presence of drainage systems.

Assessment of the resource potential of agriculture gives grounds for a significant increase in agricultural production and also for increase of its competitiveness on domestic and global markets. The main factors that determine the fuller the use of the potential available in the industry are:

- modernization and transition to innovative development model, accelerated development of modern achievements of science and technology, allowing to increase labor productivity;

\footnotetext{
*Corresponding author: rubtsov@mail.ru
} 
- creating favorable conditions for the development of IT technologies for agriculture and the implementation of pilot projects;

- popularization of modern information technologies (Internet of things) among agricultural enterprises through holding forums, etc.

Elaboration of market stimulation conditions for hardware and software systems in the following areas:

- soil control;

- control over the safety of products (Internet vegetables) - control of production growth and productivity - logistics facilities;

- control of livestock (automation of grazing and protection, monitoring of the state);

- assessment of the integration of foreign technology with domestic solutions of the Internet of things in Russia.

To introduce modern technologies of "Precision farming" it is necessary to create:

- regional centers of operative monitoring of agricultural lands on the basis of satellite, air and ground mobile systems of remote sensing;

- test areas for ground measurements, which provide rural producers and agribusiness control bodies with operational information about the state of crops and soil cover for analysis, forecasting and control decisions.

Many tasks should be solved by regional and Interdistrict centers of portal information services for the monitoring of agricultural areas.

The infrastructure of the agrarian sector (in terms of agriculture) involves navigation satellites (NS), unmanned aerial vehicles (UAVs) and tractors - robots with a set of units (TR) (Fig. 1). It should be noted that at the moment the aircraft find more acceptance than ground robotic systems $[2,3]$.

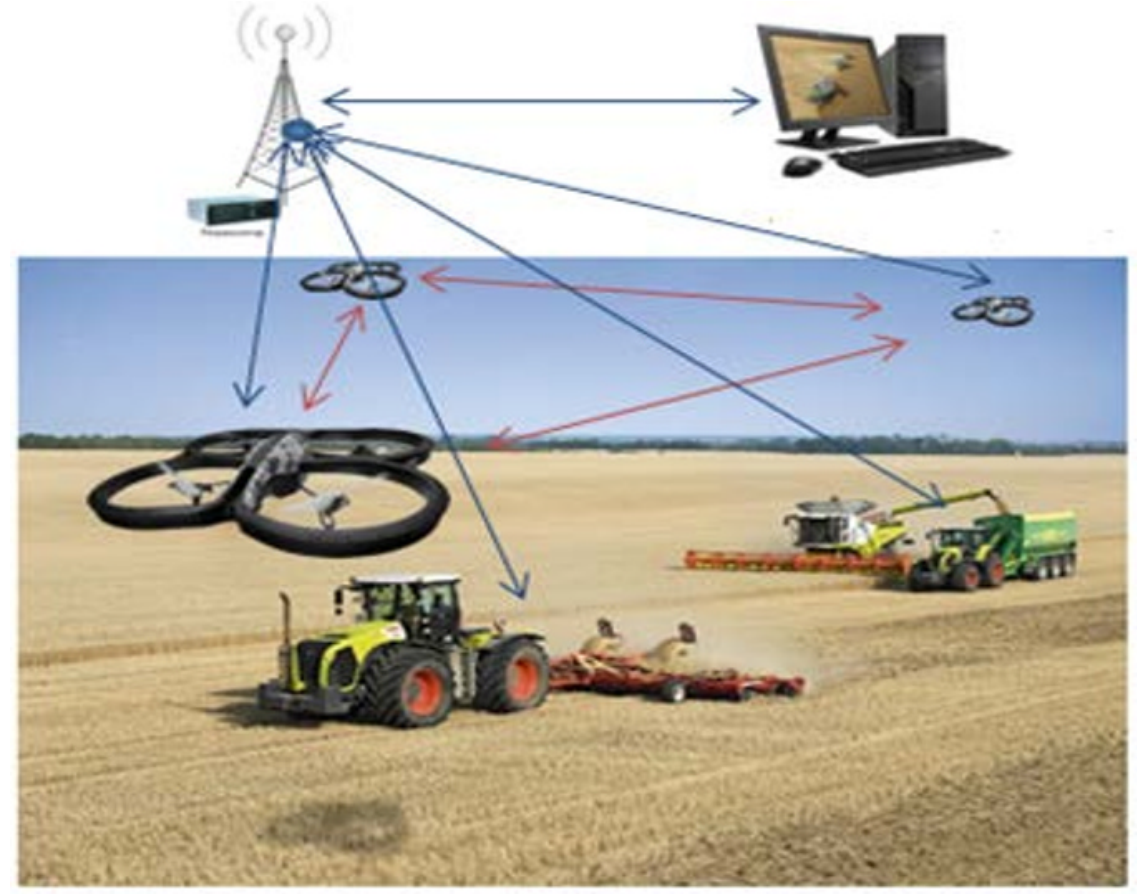

Fig.1. A heterogeneous group of service. 
Modern information technology, using large data sets with NS and UAVs and automating the collection and analysis of the information will allow developing new processes and solving a wide range of tasks in inspection and inventory of land, converting the height map into the map of the slopes, carrying out phytosanitary control, conducting the monitor.

The domestic ground component of robotics in rural owners is at an early stage of development [4]. At the present moment, when informatization is the determining vector of fundamental scientific works in the field of mechanization of agriculture, the era of the next round of agricultural production development "exact land-division" (Fig.2). The smooth transition from saturation of the producer with quality equipment, automation of processes of control, robotization of TR, and transition to regime control is obvious.

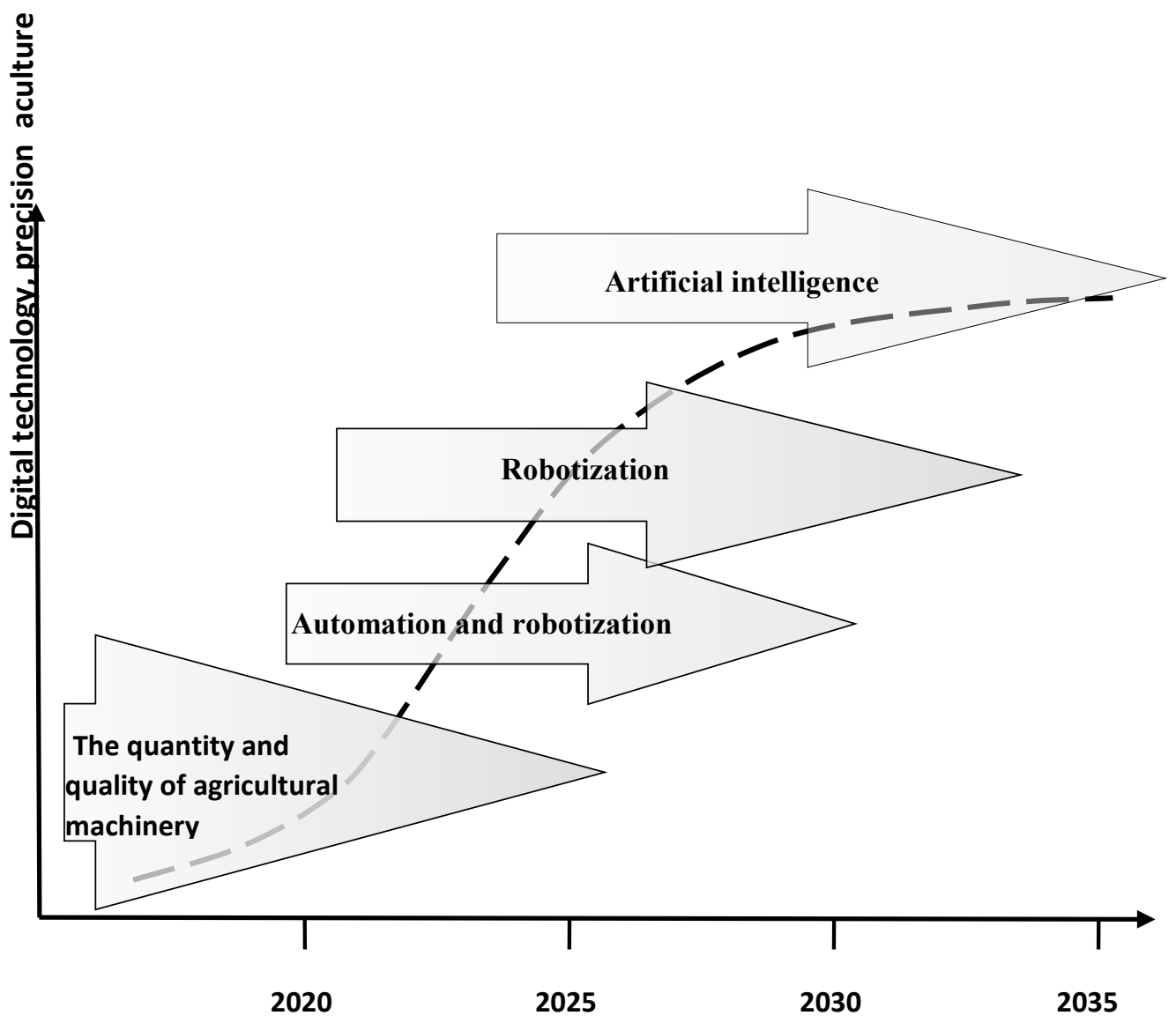

Fig. 2. Development of robotics technologies in agriculture.

Based on the results of the analysis of domestic and foreign experience in the development and use of robotic systems the main trend that determines the ways of creating promising TP is the gradual increase in the intellectual capabilities of remotely controlled samples with the gradual elimination of control functions on the part of operators.

This involves the creation of a leading scientific and technical reserve, providing increased mobility and Autonomous operation of the TR, the development of intelligent information control systems to achieve the desired degree of autonomy and group interaction for the mass use of transport robots or a number of other self-moving perspective units. It should be noted that the current practical measures for the development of domestic IT 
technologies and mobile robotics in the field of special robotics [5,6,7], create good preconditions for increasing the efficiency of agriculture.

The main vector of development of agricultural robotics is aimed at the development of innovative technologies necessary for:

- to increase the range of operation, control channels and communication;

- machine vision and navigation system improvements;

- autonomous traffic control equipment and accessories;

- automatic recognition of dynamic scenes and situations;

- creation of intelligent information processing and control systems that provide the necessary level of autonomy of transport robots for their group use.

When developing unmanned farming technologies, it is necessary to pay attention to some problem areas. For example, insufficiency of domestic element base of computing technology and electro-mechanical drives and the means of achieving the vision system in different spectral ranges. The insufficient level of technical culture in the agro-industrial complex is also should be considered.

Based on the results of the analysis of domestic and foreign experience in the development and use of robotic system the main trend that determines the ways of creating promising TP is the gradual increase in the intellectual capabilities of remotely controlled samples with the gradual elimination of control functions on the part of operators.

This involves the creation of a leading scientific and technical reserve, providing increased mobility and Autonomous operation, the development of intelligent information control systems to achieve the desired degree of autonomy and group for the successful operation of unmanned systems is necessary, from the ground up, the building of the robotic support service.

One of the most important is the solution of the issue of personnel training for the agroindustrial complex and the direction of development of scientific and information support:

The implemented measures will allow achieving the following social and economic effects:

1. To ensure the growth of labor productivity (in accordance with the strategy of socioeconomic development of the country until 2020) not less than 2 times;

2. To provide to the Russian Agrocomplex technical security at the expense of development of domestic agricultural machine building (a share not less than 70\%);

3. To raise the level of technological and technical knowledge in the industry by creating a system of innovative development, modernization of the training process as the basis for the formation of innovative agroeconomics;

4. To increase the population's access to food, to ensure the development of the territories;

5. To create conditions for effective modernization of complex machine technologies of agricultural production in Russia using information resources and automation;

6. To create new research and production companies and jobs, including with the participation of foreign firms;

7. To expand the research and production potential of small and medium enterprises in the agro-industrial sector and improve the conditions for its development.

\section{References}

1. The order of the Government of the Russian Federation, Strategy of sustainable development of rural territories of the Russian Federation for the period till 2030 (February 2, 2015) http://dokipedia.ru/document/5208837

2. I. Yu. Savin ., Y. I. Wernik, I. Paraclis, The possibility of using unmanned aerial vehicles for operational monitoring of soil productivity, 80, pp. 95-105 (2015). 
3. I. Yu. Savin, Y. I. Wernik, V. A. Isaev, Operational satellite monitoring of crops (Herald of the Russian Academy of agricultural Sciences, Moscow, 2014)

4. I. V. Rubtsov, O. G. Rusanova., Z. A. Godzhaev, The use of special-purpose technologies in agricultural robotics (Agricultural machines and technologies, 12, pp 37 - 41 (2018)

5. I. V. Rubtsov, V. S. Lapshov, K. Yu. Mashkov, V. Yu. Korchak, Robotic systems for military purposes: scientific and technical directions of development (Strategic stability, Moscow, 2016)

6. I. V. Rubtsov, A. A. Boslyakov, V. S. Lapshov, K. Yu. Mashkov, V. Noskov, Problems and prospects of development of mobile military equipment/ (BMSTU, Moscow, 2015)

7. V. I. Rubtsov, K.Yu. Mashkov., V. Yu. Korchak, Problematic issues of development of ground military robotics (Strategic stability, Moscow, 2017) 